\title{
Exploratory Data Analysis for Investigating GC-MS Biomarkers
}

\author{
Ken McGarry ${ }^{1}$, Kim Bartlett ${ }^{1}$, and Morteza Pourfarzam ${ }^{2}$ \\ ${ }^{1}$ School of Pharmacy, City Campus, University of Sunderland, SR1 3SD, UK \\ ${ }^{2}$ Royal Victoria Infirmary, Department of Clinical Biochemistry, \\ Newcastle Upon Tyne, NE1 4LP, UK
}

\begin{abstract}
The detection of reliable biomarkers is a major research activity within the field of proteomics. A biomarker can be a single molecule or set of molecules that can be used to differentiate between normal and diseased states. This paper describes our methods to develop a reliable, automated method of detecting abnormal metabolite profiles from urinary organic acids. These metabolic profiles are used to detect Inborn Errors of Metabolism (IEM) in infants, which are inherited diseases resulting from alterations in genes that code for enzymes. The detection of abnormal metabolic profiles is usually accomplished through manual inspection of the chromatograms by medical experts. The chromatograms are derived by a method called Gas Chromatography Mass Spectrometry (GC-MS). This combined technique is used to identify presence of different substances in a given sample. Using GC/MS analysis of the urine sample of the patient, the medical experts are able to identify the presence of metabolites which are a result of an IEM.
\end{abstract}

\section{Introduction}

The recent advances in bio-medical screening technologies has enabled the development of the so-called "omics" fields, such as genomics (the study of genes sequences and their regulatory mechanisms); transcriptomics (RNA and gene expression); proteomics (protein expression) and metabolomics (metabolites and metabolic networks). In this paper we are primarily concerned with the generation and analysis of metabolomic data for the early diagnosis of disease. The motivation to diagnose disease states through the use of a non-invasive technique (avoids the need for surgery) such as profiling blood or urine samples for their metabolic contents is highly attractive and desirable. It is evident that metabolomics reflects the operation of the cell since each metabolite is the result of a bio-chemical process.

The main idea behind metabolic profiling is the identification of unique signal peaks to differentiate between two or more groups such as normal and diseased samples. Each peak represents the presence of a metabolite and the expectation is that the presence/absence of certain peaks is indicative between the groups. Although, the search for such biomarkers is highly motivated, there are many difficulties; most studies are performed on small sample sizes (20-100 samples)

M. Chetty, A. Ngom, and S. Ahmad (Eds.): PRIB 2008, LNBI 5265, pp. $349-358,2008$.

(C) Springer-Verlag Berlin Heidelberg 2008 
and contain complex mixtures of metabolites common to all samples. Furthermore the small sample sizes often lead to the "curse of dimensionality" problem so often encountered in computational statistics and machine learning [1].

The study reported in this paper is concerned with the identification of inborn errors of metabolism in infants. The errors are due to a faulty gene that encodes an enzyme which translates or transports a biological product into another [2] A given IEM (there are several) will lead to a build up of a particular product which is invariably toxic [3]. Early detection of such conditions is critical since they lead to a number of highly delibating conditions symptoms in babies from poor feeding, vomiting and in older children can lead to autism, learning difficulties and mental retardation. Many IEM's can be treated with drugs and special diets. Fortunately, such conditions are rare, the occurrences of each IEM vary but perhaps they affect 1 in 5,000 individuals. GC-MS was first applied to identify diagnostic marker for IEMs by Tanaka [4. Since then GC-MS has been widely used for separation of complex biological mixtures and identification of their components, and has greatly contributed to the study and characterizations of IEM's 5667 .

The aim of this work is to develop a hybrid intelligent system using various pattern recognition and machine learning algorithms which could potentially identify all known IEMs and also be able to state whether a given sample is normal i.e. all metabolite profiles present in the sample are results of a normal metabolism or if the sample has any abnormal metabolites present in it which could then be further analysed by medical experts to see if the abnormal metabolites are the cause of the problems in the patient.

The remainder of this is paper is structured as follows; section two discusses GC-MS data issues and biomarker detection; section three describes the data preprocessing issues specific to GC-MS problems and the computational techniques we use; section four presents related work; section five highlights the experimental setup and our results; finally section six presents the conclusions.

\section{GC-MS Data Issues and Biomarker Detection}

Metabolomics is one of the most promising technologies developed so far for the analysis of living systems. It is essentially a two-stage process with the GC (Gas Chromatography) stage using a capillary column to separate the molecules, this depends on time according to the molecular weight detecting the time at which a metabolite is released (retention time) and its relative abundance appearance). 8]. At the next we use the MS (Mass-Spectrometer) which breaks each molecule down into a set of fragments (peptides), each one has set of mass/charge ratios in which the peaks correspond to the chemical composition of the metabolites, which enables identification 91011]. Combining the GC data with the MS data prevents metabolite identification problems which can often occur when the using GC or MS separately. GC-MS equipment is now a popular choice in many labs 


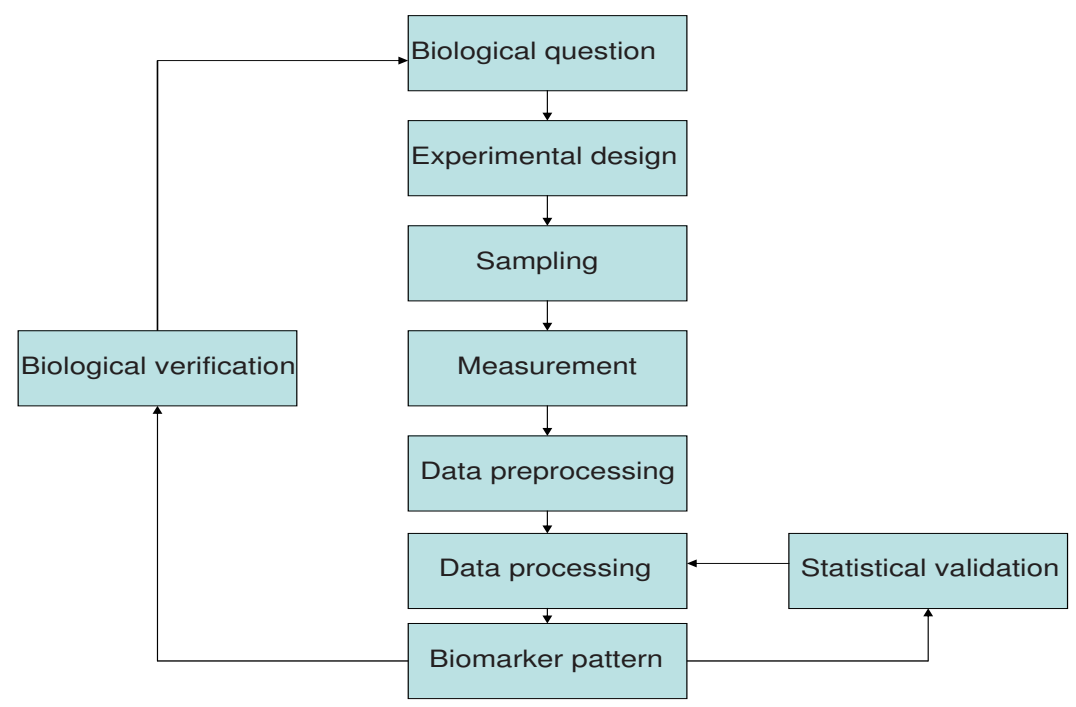

Fig. 1. The stages of biomarker detection (from Smit 2008)

worldwide because their cost have fallen in recent years but their accuracy and reliability has increased.

Irrespective of the hypernated mass spectra techniques used e.g. GC-MS, HPLC, CE-MS, or LC-MS they all suffer from the same difficulties. The main problem is the "curse of dimensionality" [12. GC-MS is considered as one of the best available methods for forensic substance identification, but the data which is generated is one of very high dimensionality. Fortunately, there is a great deal of redundancy which can be partly managed by data reduction methods such as PCA and ICA. Automated analysis of this data for presence of metabolites is difficult because of this high-dimensionality and other complexities of the data; therefore the search for reliable biomarkers is of great importance [1314]. A typical workflow model is presented in figure 1, here several stages are required, with data preprocessing playing a major part [15].

As with most data mining endeavors, the data cleansing and preprocessing stage consumes the majority of the effort [16]. Furthermore, if mass-spectra data are to be analysed, then considerable care must be taken to align the spectrograms 1718 . This was not an issue in the study described in this paper, as the authors are dealing only with the GC-chromatograms, although they are similar visually, in the sense they are peaks displayed against time [19].

In table 1, some of the inborn errors of metabolism are presented (IEM). The first column is the chemical abbreviation of the disorder, the second column indicates a biomarker typically associated with its presence and the third column describes the symptoms if the error goes unrecognised and untreated. 
Table 1. Diagnostic biomarkers for inborn errors of metabolism

\begin{tabular}{|l|l|l|}
\hline IEM & Diagnostic Biomarker & Symptoms if untreated \\
\hline PKU & Phenylalanine hydroxylase - PHE & mental retardation, autistic behavior \\
MCADD & medium chain acyl CoA dehydrogenase - C8 & fasting intolerance, hypoglycemia \\
$3-M C C D$ & 3-Methylcrotonyl CoA carboxylase deficiency & metabolic acidosis and hypoglycemia \\
SCAD & short-chain acyl-Coa dehydrogenase & $\begin{array}{l}\text { austic behaviour, mental retardation, } \\
\text { impairment of neurophysical development }\end{array}$ \\
\hline
\end{tabular}

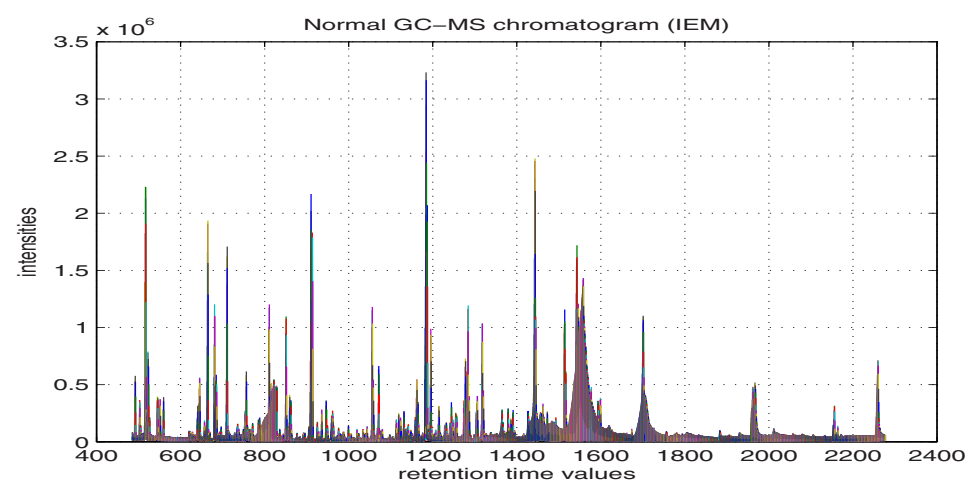

Fig. 2. GCMS Normal total chromatogram

\section{Data Preprocessing and Analysis}

The data were generated by a Agilent $6890 \mathrm{~N}$ GC coupled to a mass selective detector and an Agilent ChemStation was used. Helium was used as carrier gas with an average linear velocity of $37 \mathrm{~cm} / \mathrm{s}$. Inlet pressure was $8.44 \mathrm{psi}$. The injector split ratio was set to 1:40. Injector and interface temperatures were set $265 \mathrm{C}$ and $300 \mathrm{C}$ respectively. Samples were analysed using temperature programming (4min isothermal at $65 \mathrm{C}, 6 \mathrm{C} / \mathrm{min}$ to $275 \mathrm{C}$ followed by $2 \mathrm{~min}$ isotheraml at $275 \mathrm{C}$ ). The mass spectra were acquired over the range $40-550 \mathrm{~m} / \mathrm{z}$ at $3 \mathrm{scans} / \mathrm{min}$. The total run time was $38 \mathrm{~min}$. The data set is composed of 22 normal samples and 13 abnormal samples and is in the netCDF le format. The data were converted from netCDF format into Matlab structures using the SNCTools package.

Principal components analysis (PCA), is useful in high-throughput proteomics experiments because of the intrinsic redundancy of the data. PCA involves the transformation of the original dataset into a smaller subset with fewer variables that are uncorrelated. The principal components identify where the maximum variance of the data occurs, each component is an axis in multidimensional space.

Equation 1 describes the first principal component of the data, $y_{1}$ is the linear combination.

$$
y_{1}=a_{11} x_{1}+a_{12} x_{2}+\ldots+a_{1 p} x_{p}
$$


Equation 22 describes the second principal component of the data, and the variance is constrained by $\mathbf{a}_{\mathbf{2}}^{\prime} \mathbf{x}$ which ensures that $y_{1}$ and $y_{2}$ are uncorrelated.

$$
y_{2}=a_{21} x_{1}+a_{22} x_{2}+\ldots+a_{2 p} x_{p}=\mathbf{a}_{\mathbf{2}}^{\prime} \mathbf{x}
$$

Prior to using PCA, we normalised the data by dividing each variable by its standard deviation. Normalisation is usually good practice, it is essential in this application because the variances of the raw data are considerable.

We used the princomp function from the Matlab statistics toolbox to process the data from the original 34 (samples) x 5221 (data elements) to 34 x 20 matrix. Figure 4 shows the Pareto chart with a scree plot of the percent variability explained by each principal component, for the first 10 principal components. The 1 st component accounts for only $18 \%$ of the variance, and the next 9 components each account for perhaps 10-6\% of the remaining variation.

Plotting the two main principal components, as shown in figure 5. we obtain the familiar horseshoe shape. The biplot function can help to visualize both the

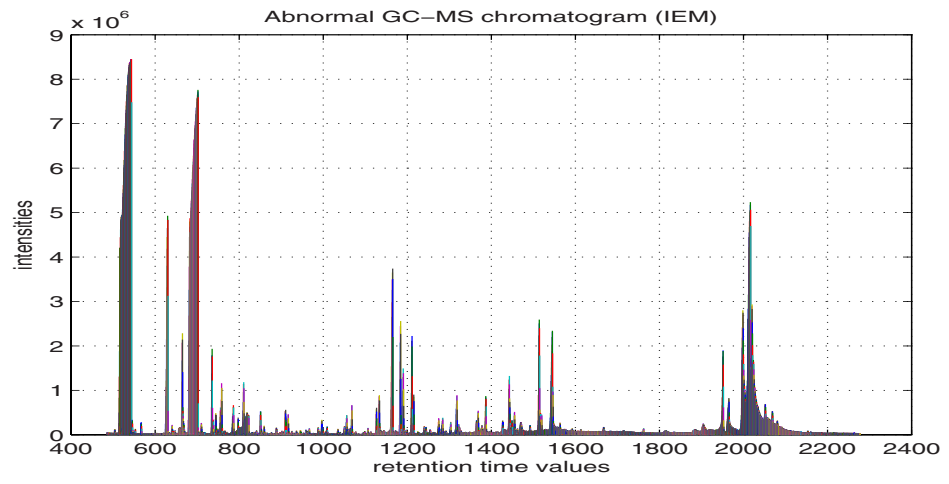

Fig. 3. GCMS Abnormal total chromatogram

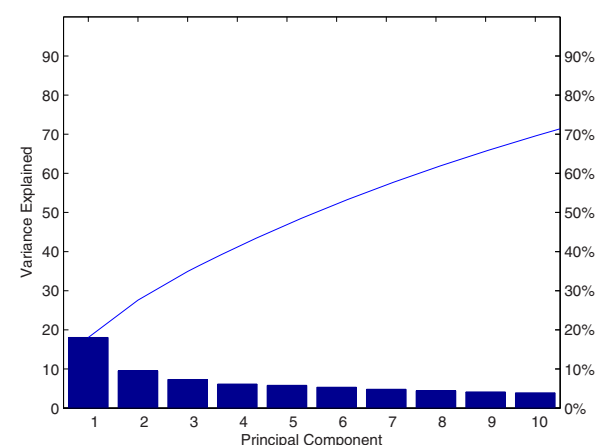

Fig. 4. Pareto diagram showing of the variances explained by the principal components

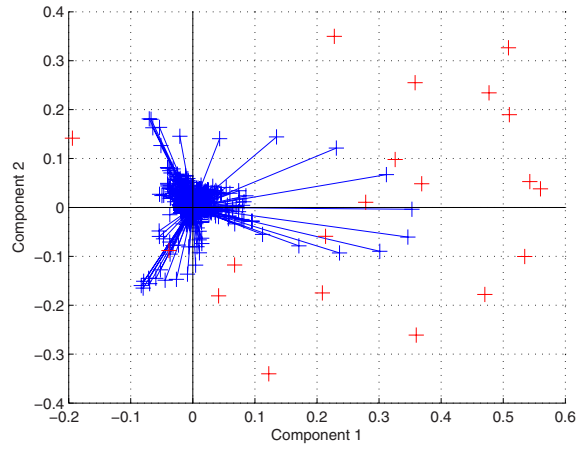

Fig. 5. Biplot principal component coefficients for each variable and the principal component scores for each observation 


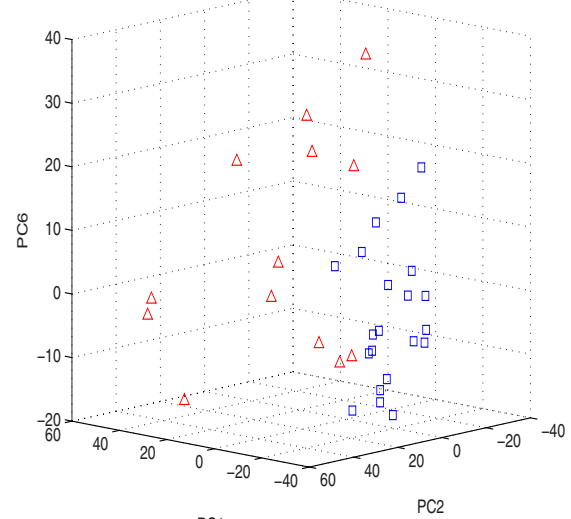

PC1

Fig. 6. K-means clustering with two clusters

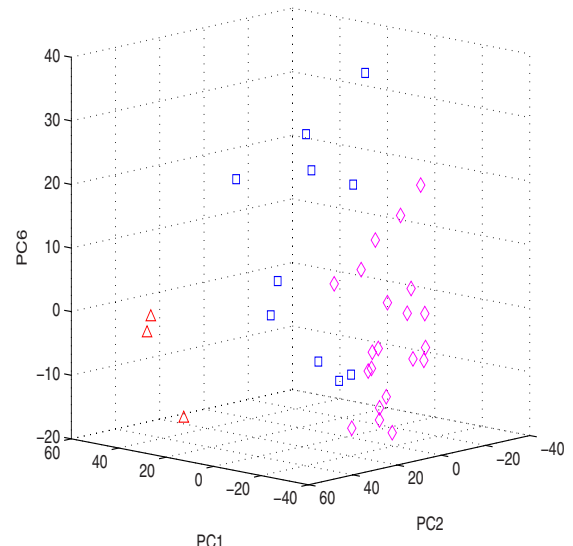

Fig. 7. K-means clustering with three clusters

principal component coefficients for each variable and the principal component scores for each observation in a unified diagram 20. The length of the lines indicates the variances of the corresponding components and the angles between them show the size of their correlations i.e. small angles indicate high correlations.

$\mathrm{K}$-means clustering was used to test the degree of discriminability between two classes (normal and abnormal), the 34 samples with 20 principal components were clustered as shown in figure 6. This shows the grouping for two clusters, which is the naturally occurring partition for our data set. We were interested in the groupings that would be formed and if each sample would be correctly allocated to the appropriate cluster. K-means clustering is a partitioning method, which operates by partitioning the objects into K mutually exclusive clusters, such that objects within each cluster are as close to each other as possible, and as far from objects in other clusters as possible.

The objective of K-means clustering is to divide the data into $k$ groups so that the within group sum-of-squares is minimized 21. The number of clusters used must be chosen in advance. We define the within-group data matrix by:

$$
\mathbf{S}_{W}=\frac{1}{n} \sum_{j=1}^{g} \sum_{i=1}^{n} I_{i j}\left(x_{i}-x_{j}\right)^{T}
$$

Where: $I i j$ is unity when $x_{i}$ belongs to group $j$ and zero if not, $g$ is the number of clusters. The sum of the diagonal elements $S_{W}$ to be minimized is given by $S_{W}=\sum S_{W_{i} j}$.

Table 2. False Positive and True Positive rates for K-means clustering (2 clusters)

\begin{tabular}{|l|l|l|l|}
\hline Abnormal TP & Abnormal FP & Normal FP & Normal TP \\
\hline $1,3,4,6,7$ & $15,18,2021$ & $1,2,5,8$ & $14,16,17,19,22,23,24,25$ \\
$9,10,1112,13$ & & & $26,27,28,29,30,31,32,33$ \\
\hline
\end{tabular}


Figure 7 shows the grouping for three clusters, it was originally thought that the misgrouped data may reside in a separate cluster. However, in both cases, a number of samples were wrongly allocated.

In table 2, we can see that certain samples were allocated to the wrong clusters. We tried two and three clusters, two clusters is the natural grouping for this data.

\section{Model Building and Experimental Results}

The initial data analysis and preprocessing appeared encouraging and confirmed that it would be worthwhile to build a classifier model to detect the difference between normal and abnormal samples. From a biological and data mining viewpoint, the most satisfying models are produced by decision trees. The decision tree method is to divide the available data using the class labels into mutually disjoint sets. During the training process, hyperplanes (decision boundaries or surfaces) are generated based upon these data-class tuples, hyperplanes (nodes) are continually added until all of the training data is accounted for.

Structurally, the completed tree is composed of leaf nodes, which correspond to important variables where numerical tests are made, the final terminal nodes correspond to the class labels. The main advantage of the decision tree model is its transparency, it is very easy to see how each class is defined in terms of the variables used and numerical tests made. The training algorithms (there are several) generally use information theory or some form of entropy calculation which tests all the variables for their information bearing potential, thus the first node (variable) is the most important, followed by tests on the remaining variables. The tree is easily converted into rules for use in an expert system.

We developed a decision tree model using 10-fold crossover validation to compute the cost vector. This process partitions the sample into 10 subsamples, each chosen randomly, however with roughly equal size. The decision trees, also have the subsamples in approximately the same class proportions. The Matlab function treefit was used, and for each subsample, a tree was fitted to the

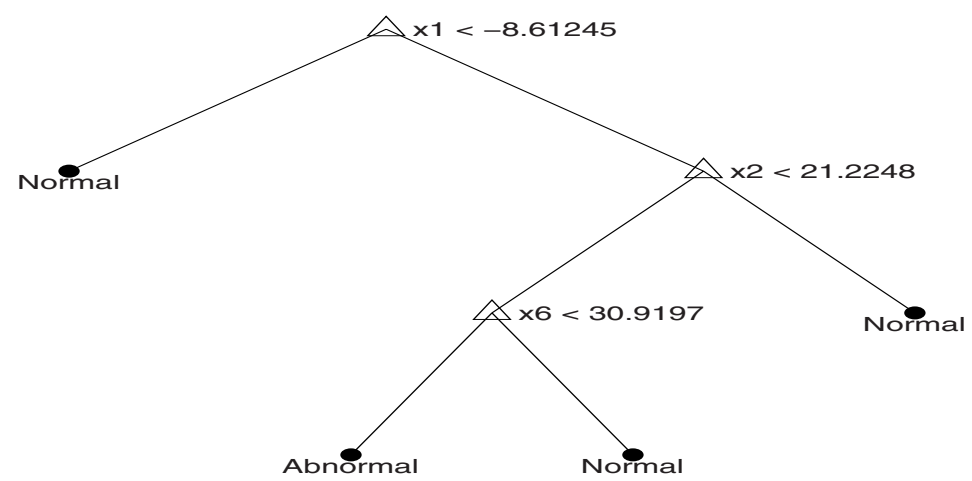

Fig. 8. Decision tree constructed with PC components 


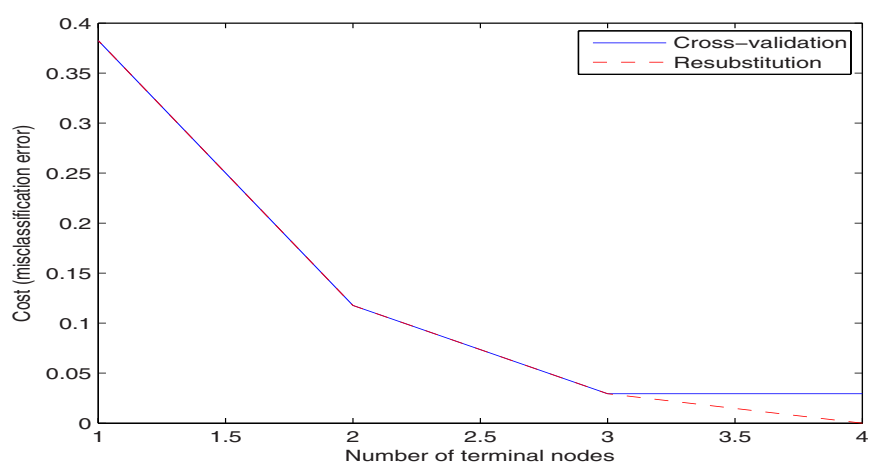

Fig. 9. Decision tree cross-validation

remaining data and then used to predict the subsample. This information is then pooled from all subsamples to compute the cost for the entire sample. The final tree is shown in figure 8 .

The results of the cross-validation are shown in figure 9] here the cost (misclassification error) is displayed against the number of nodes used within the different models developed. The optimum, model used three nodes and was 100\% accurate.

\section{Related Work}

A statistical model based on linear regression, augmented by expert rules, was demonstrated by Baumgartner et al to have highly accurate classification rate in terms of sensitivity $(>95.2 \%)$ and a very low false positive identification rate $(0.001 \%)$ [22. This research did show that regression could potentially be used to identify the disorders very accurately but their method was employed on a database of already quantified metabolites detected using Modern Tandem Mass Spectrometry (MS/MS). Further work by Baumgartner et al involved the development of a biomarker identification algorithm which improved the discriminatory powers of their earlier regression model [23].

\section{Conclusions}

The exploratory analysis conducted has revealed for this particular IEM, that the abnormal sample were extreme cases and thus easy to differentiate from the normal samples. The initial analysis using k-means clustering, had an accuracy of $77 \%$ in determing, for a two class problem how the data should be divided. The more sophisticated decision tree model was very accurate at $100 \%$, however, we expect this to drop as more IEM's are introduced into the model. Future work will involve the collection of more data from normal and abnormal sources. The 
difficulties, for data collection arise from the rarity of the abnormal cases. The intention is to build into our model more IEM's to enable a methodology for fast, automated screening.

\section{Acknowledgments}

We wish to thank the developers of the Matlab SNC Tools package.

\section{References}

1. Humphrey-Smith, I., Dracup, W.: The search for validated biomarkers in the face of biosystems complexity. Drug Discovery World, 49-56 (Spring 2005)

2. Kumps, A., Duez, P., Mardens, Y.: Metabolic, nutritional, latrogenic, and artifactual sources of urinary organic acids: a comprehensive table. Clinical Chemistry $48(5), 708-717(2002)$

3. Chu, C., Xiao, X., Zhou, X., Lau, T., Rogers, M., Fok, T., Law, L., Pang, C., Wang, C.: Metabolomic and bioinformatic analyses in asphyxiated neonates. Clinical Biochemistry 39, 203-209 (2006)

4. Tanaka, K., Budd, M., Efron, M., Isselbacher, K.: Isovaleric acidemia: a new genetic defect of leucine metabolism. Proc. Natl. Acad. Sci. USA 56(1), 236-242 (1966)

5. Kimura, M., Yamamoto, T., Yamaguchi, S.: Automated metabolic profiling and interpretation of GC/MS data for organic acidemia screening: a personal computerbased system. Journal of Experimental Medicine 188, 317-334 (1999)

6. Halket, J., Przyborowska, A., Stein, S., Mallard, W., Down, S., Chalmers, R.: Deconvolution gas chromatography/mass spectrometery of urinary organic acids potential for pattern recognition and automated identification of metabolic disorders. Rapid Communications in Mass Spectrometry 13, 279-284 (1999)

7. Ho, S., Lukacs, Z., Hoffmann, G., Linder, M., Wetter, T.: Feature construction can improve diagnostic criteria for high-dimensional metabolic data in newborn screening for medium-chain acyl-coa dehydrogenase deficiency. Clinical Chemistry 53(7), 1330-1337 (2007)

8. Duran, A., Wang, L., Yng, J., Sumner, L.: Metabolomics spectral formatting, alignment and conversion tools MSFACTS. Bioinformatics 19(17), 2283-2293 (2003)

9. Hanson, M., Andersen, B., Smedsgaard, J.: Automated and unbiased classification of chemical profiles from fungi using high performance liquid chromatograph. Journal of Microbiological Methods 61, 295-304 (2005)

10. Guillo, C., Barlow, D., Perrett, D., Hanna-Brown, M.: Micellar electrokinetic capillary chromatography and data alignment analysis: a new tool in urine profiling. Journal of Chromatography A 1027, 203-212 (2004)

11. Baran, R., Kochi, H., Saito, N., Suematsu, M., Soga, T., Nishioka, T., Robert, M., Tomita, M.: MathDAMP: a package for differential analysis of metabolite profile. BMC Bioinformatics 7, 1-9 (2006)

12. Broadhurst, D., Kell, D.: Statistical strategies for avoiding false discoveries in metabolomics and related experiments. Metabolomics 2(4), 171-196 (2006)

13. Damian, D., Oresic, M., Verheij, E., Meulman, J., Friedman, J., Adourian, A., Morel, N., Smilde, A., Van Der Greef, J.: Applications of a new subspace clustering algorithm (COSA) in medical systems biology. Metabolomics 3(1), 69-77 (2007) 
14. Goodacre, R., Vaidyanathan, S., Dunn, W., Harrigan, G., Kell, D.: Metabolomics by numbers: acquiring and understanding global metabolite data. TRENDS in Biotechnology 22(5), 245-252 (2004)

15. Smit, S., Hoefsloot, H., Smilde, A.: Statistical data processing in clinical proteomics. Journal of Chromatography B866(1-2), 77-88 (2008)

16. Obuchowshi, N., Lieber, M., Wians, F.: ROC curves in /it Clinical Chemistry: uses, misuses, and possible solutions. Clinical Chesmitry 50(7), 118-1125 (2004)

17. Leibermeister, W., Klipp, E.: Bringing metabolic networks to life: integration of kinetic, metabolic and proteomic data. Theoretical Biology and Medical Modelling 42(3), 1-15 (2006)

18. Yeang, C., Vingron, M.: A joint model of regulatory and metabolic networks. BMC Bioinformatics 332(7), 1-5 (2006)

19. Hilario, M., Kalousis, A., Prados, J., Binz, P.: Data mining for mass spectra-based cancer diagnosis and biomarker discovery. Drug Discovery Today 2(5), 214-222 (2004)

20. Gower, J., Hand, D.: Biplots. Chapman and Hall, London (1996)

21. Martinez, W., Martinez, A.: Exploratory data analysis with Matlab. Chapman and Hall, New York (2000)

22. Baumgartner, C., Bohm, C., Baumgartner, D.: Modelling of classification rules on metabolic patterns including machine learning and expert knowledge. Journal of Biomedical Informatics 38(2), 89-98 (2005)

23. Baumgartner, C., Baumgartner, D.: Biomarker discovery, disease classification, and similarity query processing on high-throughput MS/MS data of inborn errors of metabolism. Journal of Biomolecular Screening 11(1), 90-99 (2006) 